\title{
Resistance to desiccation and skin fatty acids in outbreak strains of methicillin-resistant Staphylococcus aureus
}

\author{
M. FARRINGTON, N. BRENWALD, D. HAINES and E. WALPOLE \\ Clinical Microbiology and Public Health Laboratory, Addenbrooke's Hospital, Hills Road, Cambridge CB2 $2 Q Q$
}

\begin{abstract}
Summary. Resistance to desiccation and to skin fatty acids was measured in three groups of methicillin-resistant Staphylococcus aureus (MRSA) strains and a group of control strains. Organisms from a large outbreak on a special care baby unit (SCBU), where MRSA had been isolated from staff hands but not from the environment, were significantly more sensitive to drying than strains from a burns unit where extensive environmental contamination had been demonstrated. MRSA from other wards, in the same hospital but not associated with large outbreaks, gave heterogeneous results. Fatty-acid resistance, determined by an agar dilution method, was not associated with strain origin. Some epidemic strains of MRSA were relatively sensitive to desiccation, and the abilities of such strains to spread widely on a SCBU by the hand-borne route could not be explained by enhanced resistance to skin fatty acids.
\end{abstract}

\section{Introduction}

During a large outbreak of methicillin-resistant Staphylococcus aureus (MRSA) infections at the Prince of Wales Hospital (PWH), a new 1400-bed teaching centre in Hong Kong, ${ }^{1}$ two MRSA strains distinguished by phage type and plasmid profile were predominant. One strain was common on the special care baby unit (SCBU) where hand-borne transmission was confirmed to be important and no environmental contamination could be demonstrated. The other was probably spread by various routes on the burns unit (BU), and was readily isolated from the environment. A territory-wide survey confirmed that these two strains were associated with the same types of unit in other Hong Kong hospitals. Several other apparently distinguishable strains were isolated sporadically in all units of the $\mathrm{PWH}$ without causing large outbreaks.

There are three closely related but distinct major routes for transmission of staphylococci between hospital patients, namely dissemination through the air, direct contact with organisms on equipment or in the environment, and long- or short-term colonisation of the hands of clinical attendants. Great variation in the relative importance of each route has been reported between different types of hospital unit. Thus handborne spread is predominant on SCBUs ${ }^{2}$ and transmission through the air and environment is important on $\mathrm{BUs}^{3}$.

However, much less is known of any staphylococcal characteristics that may enable individual strains to adapt to one or more of these three routes. Resistance

Received 4 April 1991 ; accepted 20 April 1991. to drying and the ability to survive on skin may be important factors. Several models have been used to investigate the resistance of staphylococci to desiccation, ${ }^{4-10}$ but survival on human skin has been studied only with methicillin-sensitive strains. ${ }^{7,11}$ Measuring the in-vitro resistance of MRSA strains to fatty acids commonly found on human skin may be a measure of their abilities to survive on the skin, ${ }^{12}$ and this manoeuvre avoids the difficulties of inoculating resistant pathogens on to human volunteers.

We have compared the resistance to drying and to skin fatty acids of the three groups of organisms from the PWH associated with (1) the SCBU, (2) the BU, and (3) other wards; a reference strain "EMRSA-1" and the Oxford strain of $S$. aureus were included as controls.

\section{Materials and methods}

\section{Strains investigated}

Table I shows the characteristics of the 13 clinical isolates of MRSA that were studied. ${ }^{1,13}$ Five strains were from babies on the SCBU, four from patients on the $\mathrm{BU}$, and four were from patients on other hospital wards apparently unconnected with each other, the SCBU or the BU. The isolates from the SCBU and $\mathrm{BU}$ were chosen to display the broadest range of typing characteristics observed in organisms from each of these units, yet all were closely linked epidemiologically. These clinical isolates had been stored on nutrient-agar slopes in screw-top 7-ml bottles in the dark at room temperature. Immediately before the present study, sensitivity to antibiotics was retested by a disk diffusion method with disks containing 
Table I. Typing characteristics, and results of fatty acid sensitivity, pigmentation and lipase production of the 13 clinical MRSA isolates investigated

\begin{tabular}{|c|c|c|c|c|c|c|c|c|}
\hline \multirow[b]{2}{*}{ Source } & \multirow{2}{*}{$\begin{array}{l}\text { Strain } \\
\text { no. }\end{array}$} & \multirow{2}{*}{$\begin{array}{l}\text { Plasmid content } \\
\text { (Mda) }\end{array}$} & \multirow[b]{2}{*}{ Phage type } & \multirow{2}{*}{$\begin{array}{l}\text { Resistance } \\
\text { pattern }\end{array}$} & \multicolumn{2}{|c|}{$\mathrm{MIC}(\mathrm{mg} / \mathrm{L})$ of } & \multirow[b]{2}{*}{ Pigment } & \multirow[b]{2}{*}{ Lipase } \\
\hline & & & & & $\begin{array}{l}\text { Linoleic } \\
\text { acid }\end{array}$ & $\begin{array}{l}\text { Linolenic } \\
\text { acid }\end{array}$ & & \\
\hline $\mathrm{SCBU}$ & $\begin{array}{l}1 \\
2 \\
3 \\
4 \\
5\end{array}$ & $\begin{array}{l}\text { L } 2 \cdot 8 \\
\text { L } 2 \cdot 8 \\
\text { L } 2 \cdot 8 \\
\text { L } 2 \cdot 8 \\
\text { L } 2 \cdot 8\end{array}$ & $\begin{array}{l}77 / 932 \\
29 / 77 / 932 \\
29 / 77 / 932 \\
29 / 77 / 932 \\
77 / 932\end{array}$ & $\begin{array}{l}\text { TeGE } \\
\text { TeGE } \\
\text { GN } \\
\text { TeGE } \\
\text { TeG }\end{array}$ & $\begin{array}{l}0.03 \\
0.03 \\
0 ; 03 \\
0.03 \\
0.03\end{array}$ & $\begin{array}{l}0.03 \\
0.06 \\
0.06 \\
0.03 \\
0.03\end{array}$ & $\begin{array}{l}\text { Buff } \\
\text { Orange } \\
\text { White } \\
\text { Orange } \\
\text { Orange }\end{array}$ & $\begin{array}{l}+ \\
+ \\
- \\
+ \\
+\end{array}$ \\
\hline $\mathrm{BU}$ & $\begin{array}{l}6 \\
7 \\
8 \\
9\end{array}$ & $\begin{array}{l}\text { L } 1.71 .61 .35 \\
\text { L } 1.71 .6 \\
\text { L } 1.71 .35 \\
\text { L } 1.6\end{array}$ & $\begin{array}{l}618 / 620 \\
620 \\
\text { NT } \\
\text { NT }\end{array}$ & $\begin{array}{l}\text { TeGNE } \\
\text { TrTeGNEC } \\
\text { TeE } \\
\text { TeGNEC }\end{array}$ & $\begin{array}{l}0.015 \\
0.03 \\
0.0075 \\
0.03\end{array}$ & $\begin{array}{l}0.015 \\
0 \cdot 03 \\
0.03 \\
0.03\end{array}$ & $\begin{array}{l}\text { Buff } \\
\text { White } \\
\text { White } \\
\text { Orange }\end{array}$ & $\begin{array}{l}- \\
- \\
+ \\
+\end{array}$ \\
\hline Other & $\begin{array}{l}10 \\
11 \\
12 \\
13\end{array}$ & $\begin{array}{l}\text { L } 2 \cdot 81 \cdot 6 \\
\text { L } 2 \cdot 81 \cdot 55 \\
\text { L } 2 \cdot 0 \\
\text { L }\end{array}$ & $\begin{array}{l}\text { NT } \\
618 / 622 / 617 \\
\text { NT } \\
932\end{array}$ & $\begin{array}{l}\text { TrTeGEC } \\
\text { TeE } \\
\text { TeGNE } \\
\text { E }\end{array}$ & $\begin{array}{l}0.03 \\
0.03 \\
0.03 \\
0.03\end{array}$ & $\begin{array}{l}0.03 \\
0 \cdot 06 \\
0 \cdot 03 \\
0 \cdot 06\end{array}$ & $\begin{array}{l}\text { Buff } \\
\text { White } \\
\text { White } \\
\text { Buff }\end{array}$ & $\begin{array}{l}+ \\
- \\
- \\
-\end{array}$ \\
\hline
\end{tabular}

NT, non-typable by routine or extended set of phages; L, large plasmid of 28-35 Mda; Tr, trimethoprim; Te, tetracycline; G, gentamicin; $\mathrm{N}$, neomycin; E, erythromycin; $\mathrm{C}$, chloramphenicol.

methicillin, trimethoprim, tetracycline, gentamicin, neomycin, erythromycin, chloramphenicol, rifampicin and fusidic acid. No loss of resistance during storage was demonstrated in any strain. ${ }^{13}$ Our laboratory culture of the Oxford strain of $S$. aureus (NCTC 6571) and a reference strain of "EMRSA-1" (NCTC 11940) were included as controls; NCTC 11940 was tested in duplicate. These 16 organisms were randomised and coded so that determination of resistance to desiccation was performed without prior knowledge of their characteristics.

\section{Resistance to drying}

Methods for these tests were based upon those introduced by Rountree ${ }^{6}$ and modified by BeardPegler and her colleagues. ${ }^{9}$ Overnight cultures in Brain Heart Infusion Broth (Difco) of each strain were inoculated to fresh broth and incubated in air with shaking at $37^{\circ} \mathrm{C}$ for $4 \mathrm{~h}$. Cotton blanket material was cut into $20-\mathrm{mm}$ squares, washed in deionised water and sterilised by autoclaving. All textile squares were stored dry in sterile petri dishes until required. For each experiment, an inoculum of $c \cdot 10^{7}$ organisms from a 4-h broth culture was placed on eight cotton blanket squares that were then stored in separate sterile vented petri dishes in a dark cupboard at ambient temperature and humidity. Maximum and minimum temperature and relative humidity were recorded weekly. Numbers of surviving bacteria were assessed at intervals up to 79 days. Viable organisms in the extracts were counted by the method of Miles $e t$ al. ${ }^{14}$ with $5 \%$ horse blood agar as the recovery medium. The experiment was performed twice concurrently between January and April 1990. At each sampling time, the death rate $(K)$ per day after day 2 for each strain was calculated from the mean of its six viable counts from the formula;

$$
\mathrm{K}=2 \cdot 3\left(\mathbf{B}_{2}-\mathbf{B}_{\mathrm{t}}\right) / \mathbf{t}
$$

where $t=$ desiccation time in days $-2, B_{2}=\log _{10}$ mean count at day 2 , and $B_{t}=\log _{10}$ mean count after $t$ days. ${ }^{6}$ Statistical analysis of the differences between $\mathrm{K}$ values over each period of desiccation was by Student's $t$ test.

Sensitivity to linoleic and linolenic acid. Linoleic and linolenic acids were incorporated in Blood Agar Base No. 2 (Oxoid) to give final concentrations of $2 \%, 1 \%$, $0.5 \%, 0.25 \%, 0.125 \%, 0.0625 \%, 0.0312 \%, 0.0156 \%$, $0.0075 \%$ and $0.00375 \% \mathrm{v} / \mathrm{v}$. The technique followed that of Lacey and Lord, ${ }^{12}$ except that the endpoint was taken as the concentration of fatty acid that completely inhibited growth of an inoculum containing $10^{4}$ organisms.

Pigment and lipase production. Colonial colour and lipase activity on milk agar were observed after incubation in air for $48 \mathrm{~h}$ at $37^{\circ} \mathrm{C}$ followed by $48 \mathrm{~h}$ at room temperature. Milk agar was made by adding $50 \mathrm{ml}$ of Nestlé's Ideal ${ }^{\circledR}$ evaporated milk to $250 \mathrm{ml}$ of blood agar base no. $2{ }^{7}$

\section{Results}

\section{Resistance to drying}

Mean viable counts for different strains varied between $5.0 \times 10^{6}$ and $1.2 \times 10^{8}$ at day $2,3.6 \times 10^{6}$ and $4.8 \times 10^{8}$ at day 7 , and $1.5 \times 10^{2}$ and $3.8 \times 10^{5}$ at day 79. Thus, some strains continued to multiply between days 2 and 7, and this was a consistent property of these strains in both experiments. Figs. 1 and 2 show the death rate $(\mathrm{K})$ of each group of strains, and table 


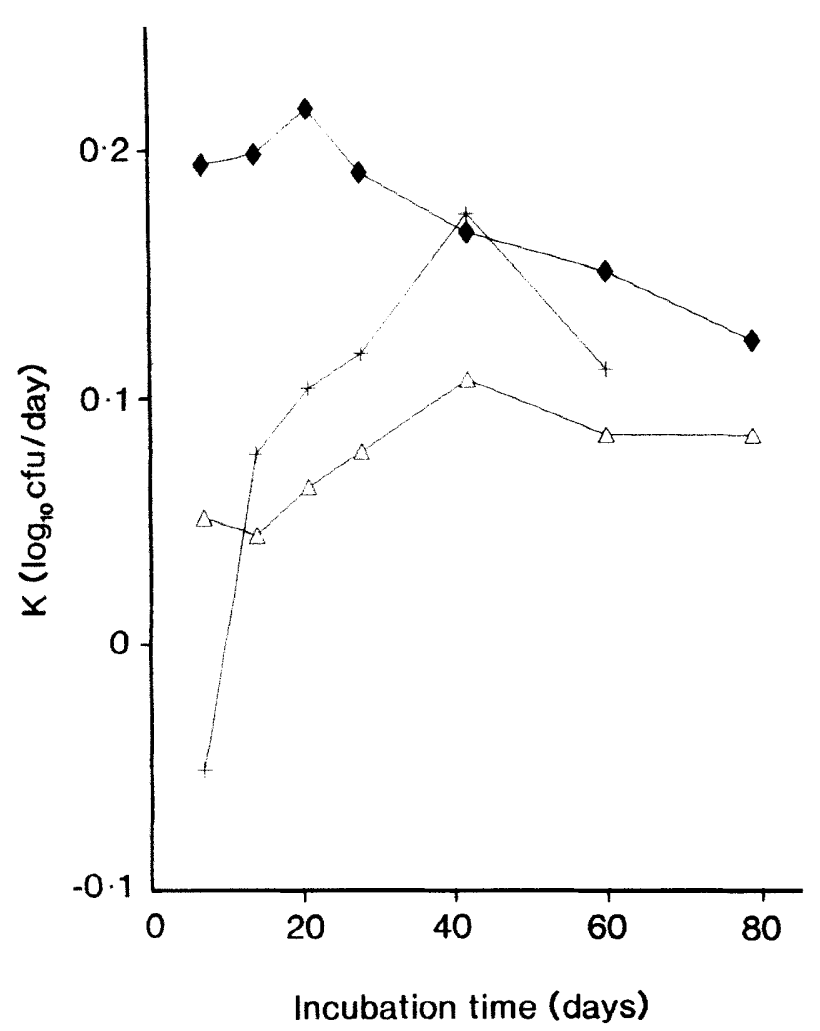

Fig. 1. Death rate $(K)$ of MRSA strains from the $\operatorname{SCBU}(\bullet), B U$ $(\triangle)$, and other wards $(+)$ during various incubation periods.

II shows the mean values of $\mathrm{K}$ and their SDs for the groups of strains associated with the SCBU and BU. The mean $\mathrm{K}$ of the strains from the $\mathrm{BU}$ was significantly lower than that of strains from the SCBU at the $5 \%$ level over $14,42,60$ and 79 days, and at the $1 \%$ level over 21 and 28 days. $K$ values for the Oxford $S$, aureus strain and the reference EMRSA-1 strain (fig. 2) were similar to those of the BU isolates, particularly for incubation times greater than 28 days (fig. 1), whereas the strains from "other" wards showed the most variable $K$ values, compared with each other and over the time period studied. The SD of the $\mathrm{K}$ values for the four strains comprising this group was the highest or second highest of all the groups at each sampling time. Organisms in the BU and EMRSA-1 groups had approximately constant $K$ values throughout the experiment, whereas the death rate of the SCBU strains showed a downward trend, although

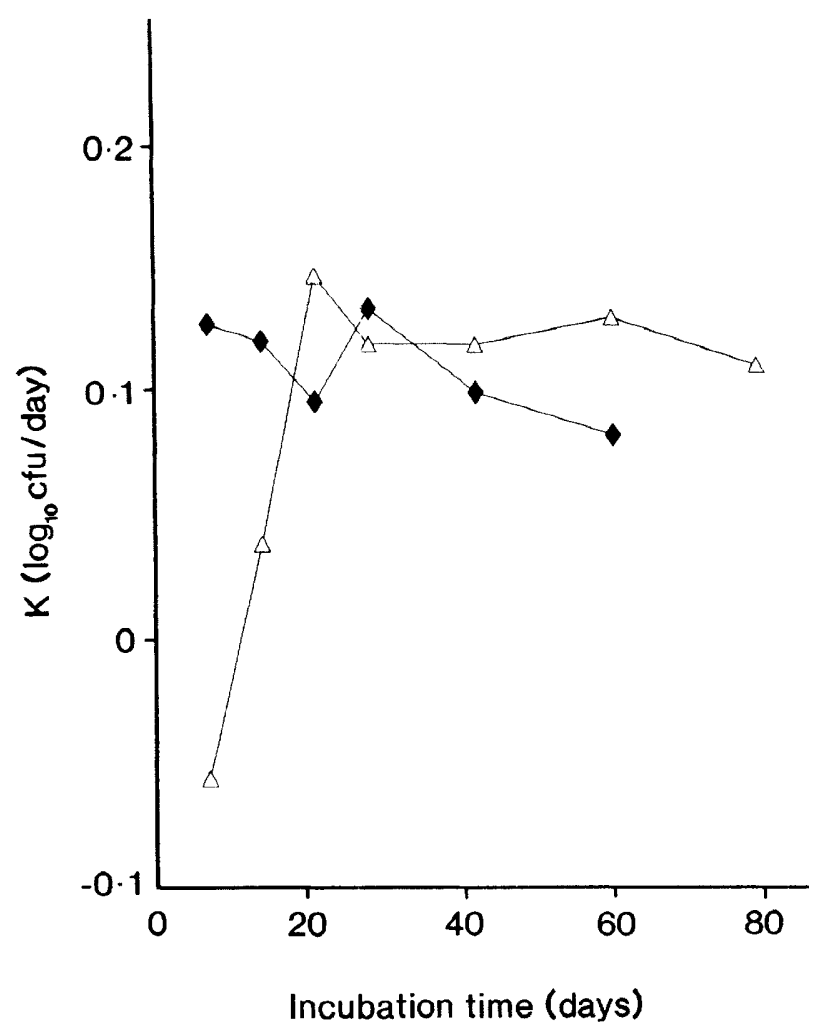

Fig. 2. Death rate (K) of EMRSA-1 ( $)$ and the Oxford S. aureus $(\triangle)$ control strains over various incubation periods.

remaining significantly higher than for strains from the $\mathrm{BU}$, and several of the non-epidemic-associated strains multiplied at the beginning of the study.

During the study, the ambient temperature was 22$25^{\circ} \mathrm{C}$ and the relative humidity was $42-54 \%$.

Sensitivity to fatty acids. Table I shows the MICs of linoleic and linolenic acid for the clinical isolates. MICs of linoleic and linolenic acid for the EMRSA-1 and the Oxford $S$. aureus strains were 0.03 and $0.03 \mathrm{mg} / \mathrm{L}$, and 0.15 and $0.03 \mathrm{mg} / \mathrm{L}$, respectively. There was a narrow range of MICs, and no obvious correlation between fatty-acid sensitivity and strain origin.

Pigment and lipase production. Colonial colour and lipase production of the MRSA strains from the PWH are shown in table I. There was no absolute relationship between pigmentation, lipase production and strain origin, although all but one strain from the SCBU

Table II. Death rates (values of K) during desiccation of strains from SCBU and BU

\begin{tabular}{|c|c|c|c|c|c|c|c|}
\hline \multirow{2}{*}{ Origin } & \multicolumn{7}{|c|}{ Mean (SD) value of $K$ after incubation for (days) } \\
\hline & 7 & 14 & 21 & 28 & 42 & 60 & 79 \\
\hline & $\begin{array}{c}0.194 \\
(0.186)\end{array}$ & $\begin{array}{c}0.199 \\
(0.094)\end{array}$ & $\begin{array}{c}0.217 \\
(0 \cdot 070)\end{array}$ & $\begin{array}{c}0.191 \\
(0.046)\end{array}$ & $\begin{array}{c}0.167 \\
(0.034)\end{array}$ & $\begin{array}{c}0 \cdot 150 \\
(0.040)\end{array}$ & $\begin{array}{c}0.121 \\
(0.027)\end{array}$ \\
\hline BU & $\begin{array}{c}0.051 \\
(0.534)\end{array}$ & $\begin{array}{c}0.044^{*} \\
(0.027)\end{array}$ & $\begin{array}{l}0.063^{* *} \\
(0.025)\end{array}$ & $\begin{array}{c}0.077^{* *} \\
(0.026)\end{array}$ & $\begin{array}{c}0.107^{*} \\
(0.032)\end{array}$ & $\begin{array}{c}0.084^{*} \\
(0.017)\end{array}$ & $\begin{array}{c}0.083^{*} \\
(0.015)\end{array}$ \\
\hline
\end{tabular}

Difference between mean values of $K$ for SCBU and BU isolates: ${ }^{*} p<0.05 ;{ }^{* *} p>0.01$. 
were pigmented, and all strains that produced orange pigment were lipase positive. Both the Oxford $S$. aureus and EMRSA-1 strains formed white, lipasenegative colonies.

\section{Discussion}

MRSA strains that spread on a burns unit through the air, the environment and staff contacts were significantly more resistant to desiccation than were strains common on a neonatal unit where hand-borne transmission was predominant. Hence, resistance to drying is not an invariable characteristic of "epidemic" staphylococci, but strains that possess it may be well fitted for survival and transmission on particular wards.

We chose desiccation conditions that approximated in some respects to those that might be experienced by organisms shed in a hospital ward, but there are many possible extrinsic determinants of dry survival that may vary even between different areas of the same room. These include the initial culture medium and growth phase, inoculum size and density, humidity, ultraviolet light intensity, temperature, and structure of the substrate.

The survival of $S$. aureus in dust from hospital wards reduces with increasing relative humidity and ultraviolet radiation. ${ }^{4,5}$ Other workers have studied the resistance to drying of laboratory cultures of staphylococci in various in-vitro and in-vivo models, and have correlated the results with phenotypic and epidemiological strain characteristics. $7,8,15$ When studying large inocula applied to cotton lint material and stored in the dark for up to 93 days, Rountree 6 found that some epidemic-associated group I methicillin-sensitive $S$. aureus (MSSA) strains survived for longer than group II isolates that had not caused epidemics in hospitals. Survival was greater when woollen blanket was used as the substrate. Epidemicassociated MRSA and MSSA strains were shown by Beard-Pegler et al. ${ }^{9}$ to have about equal resistance to desiccation on cotton blanket material after storage for 7 days, but sporadically-isolated MRSA strains were more sensitive. Over longer periods, the epidemic MRSA strains emerged as more resistant than MSSA strains. The epidemic MRSA strain produced a goldorange pigment, but pigmentation was not correlated with resistance to drying in MSSA strains.

Generally, the death rates of strains from the PWH were lower than those reported by Beard-Pegler et al. ${ }^{9}$ This may have been due to small variations in the methods used or to strain differences, but probably more important were the higher mean ambient temperature and relative humidity of Sydney compared with those of Cambridge. In support of this, rapid drying of staphylococcal inocula within $24 \mathrm{~h}$ was found by both groups in Sydney, ${ }^{6,9}$ whereas multiplication of some organisms occurred in Cambridge between days 2 and 7 . In cooler climates, more consistent early results would probably be obtained by placing the inoculated cotton blanket squares in a desiccator for the first $24 \mathrm{~h}$.

We found that the viability of most "epidemic" MRSA (those from the BU, and the EMRSA-1) remained approximately constant throughout the sampling period, but the "epidemic" strain from the SCBU died quickly in the first 6 weeks of desiccation. However, Rountree ${ }^{6}$ found that most "epidemic" strains had little loss of viability in the first 14 days of storage. This difference could also be due to strain variation, or to slow evaporation of broth from the inoculum delaying the onset of desiccation in Cambridge. Inocula of isolates from the SCBU may consist of a high proportion of desiccation-sensitive cocci and a smaller number of desiccation-resistant ones.

Duckworth and Jordans ${ }^{10}$ found the Oxford $S$ aureus to be exquisitely sensitive to drying on formica at $37^{\circ} \mathrm{C}$, but by our technique the organism showed resistance equivalent to that of epidemic MRSA isolates from the $\mathrm{BU}$. This may have been due to technical differences, or may reflect the variety of organisms that are called the "Oxford staphylococcus" in different laboratories in the UK (Dr B. D. Cookson, personal communication). Rountree also found that laboratory-adapted staphylococci were very sensitive to drying. ${ }^{6}$ It is valuable to include controls in desiccation experiments to allow inter-laboratory comparisons, and the Oxford $S$. aureus may not be reliable unless freshly obtained from a culture collection. A standard strain such as NCTC 11940 may be more typical of epidemic-associated members of the species, but this strain has also undoubtedly been repeatedly subcultured in the past.

There was no obvious correlation between resistance to desiccation and production of pigment or lipase. It is possible that the genetic determinants of the latter characteristics had been lost from some strains during storage ${ }^{15}$ but those of desiccation resistance were maintained and were, therefore, distinct in the isolates we studied.

Skin fatty acids are derived from the activity of bacterial lipases on triglycerides secreted in sebum. Linoleic and linolenic acids, found on normal human skin, are among the fatty acids with the greatest activity against $S$. aureus. ${ }^{12}$ Strains from the SCBU, where survival on hands was presumably important, were not more resistant than those from elsewhere, or than controls, by the methods we used. Others have also found only a narrow range of sensitivity of staphylococci to skin fatty acids. ${ }^{16}$ Inter-strain differences may be more readily demonstrated by other techniques, such as viable-counting on solid media. ${ }^{17}$ For example, this technique, and that of Mortimer $e t$ al. ${ }^{18}$ have shown that strains of Streptococcus pyogenes from skin infections are more resistant to skin fatty acids than those from the throat. However, the contribution of fatty acids in clinical conditions is uncertain; because skin was found to have greater anti-staphylococcal activity than glass only under 
occlusive dressings. ${ }^{7}$ A valid model to compare the survival of resistant staphylococcal strains on human skin has not yet been developed, and the apparent propensity of MRSA strains from Hong Kong neonatal wards to spread in such units remains unexplained.

\section{References}

1. Farrington $M$, Ling, J, Ling T, French GL. Outbreaks of infection with methicillin-resistant Staphylococcus aureus on neonatal and burns units of a new hospital. Epidemiol Infect 1990; 105: 215-228.

2. Mortimer, EA, Wolinsky E, Gonzaga AJ, Rammelkamp $\mathrm{CH}$. Role of airborne transmission in staphylococcal infections. Br Med J 1966; 1: 319-322.

3. Hambraeus A. Spread of Staphylococcus aureus in a burns unit. Acta Universitas Uppsaliensis: Abstracts of Uppsala Dissertations from the Department of Medicine. 1973; 158: $5-23$.

4. Lidwell OM, Lowbury EJ. The survival of bacteria in dust. II The effect of atmospheric humidity on the survival of bacteria in dust. $J$ Hyg $1950 ; 48 ; 21-27$.

5. Lidwell OM, Lowbury EJ. The survival of bacteria in dust. IV. Atmospheric humidity and the bactericidal action of ultraviolet irradiation. J Hyg $1950 ; 48: 38-43$.

6. Rountree PM. The effect of desiccation on the viability of Staphylococcus aureus. J Hyg $1963 ; 61$ : 265-272.

7. Lacey RW, Alder VG, Gillespie WA. The survival of Staphylococcus aureus on human skin: an investigation using mixed cultures. Br J Exp Pathol 1970; 51 : 305-313.

8. Lacey, RW. Effect of antibiotic resistance on the survival of Staphylococcus aureus. J Clin Pathol 1972; 25: 713-715.

9. Beard-Pegler MA, Stubbs E, Vickery AM. Observations on the resistance to drying of staphylococcal strains. $J$ Med Microbiol 1988; 26: 251-255.

10. Duckworth GJ, Jordens JZ. Adherence and survival properties
We are grateful to Dr R. Marples, Staphylococcus Reference Laboratory, Central Public Health Laboratory, London for phage typing, and to Professor G. L. French, Department of Microbiology, Guy's Hospital, London and Dr D. F. J. Brown, Clinical Microbiology and Public Health Laboratory, Addenbrooke's Hospital, Cambridge for helpful advice. of an epidemic methicillin-resistant strain of Staphylococcus aureus compared with those of methicillin-sensitive strains. J Med Microbiol 1990; 32: 195-200.

11. Filho PPG, Stumpf M, Cardoso CL. Survival of gram-negative and gram-positive bacteria artificially applied on the hands. J Clin Microbiol $1985 ; 21$ : 652-653.

12. Lacey RW, Lord VL. Sensitivity of staphylococci to fatty acids: novel inactivation of linolenic acid by serum. $\mathrm{J} \mathrm{Med}$ Microbiol $1981 ; 14: 41-49$

13. French GL, Ling J, Ling T, Hui YW. Susceptibility of Hong Kong isolates of methicillin-resistant Staphylococcus aureus to antimicrobial agents. $J$ Antimicrob Chemother $1988 ; 21$ : $581-588$.

14. Miles AA, Misra SS, Irwin JO. The estimation of the bactericidal power of the blood. $J$ Hyg 1938 ; 38: 732-749.

15. Grinsted J, Lacey RW. Ecological and genetic implications of pigmentation in Staphylococcus aureus. J Gen Microbiol 1973; 75: 259-267.

16. McDonald MI, Graham I, Harvey KJ, Sinclair A. Antibacterial activity of hydrolysed linseed oil and linolenic acid against methicillin-resistant Staphylococcus aureus. Lancet 1981 ; 2 : 1056.

17. Davidson C, Rogers C, Taylor AE, Brown DFJ, Naylor GRE Qualitative and quantitative methods of studying the effect of lipids on bacteria grown on the surface of solid culture media. J Med Microbiol 1980; 13: 469-475.

18. Mortimer GE, Pinney A, Widdowson JP. The survival of "skin" and "throat" streptococci in peptone water-oleic acid and their growth in simple peptone media. In: Parker MT (ed) Pathogenic streptococci. Chertsey, Reedbooks 1979: 120-121. 